\title{
THE CENTRE OF EARTHQUAKE LOADING ON TALL BUILDINGS
}

\author{
H. M. Irvine*
}

\begin{abstract}
A response spectrum technique is applied to two well known linear elastic continuous models of slender high rise buildings in order to obtain likely positions for the centres of earthquake loading. The models chosen are the uniform cantilever shear beam and the uniform cantilever shear wall, which represent the two extremes of pure shear deformation and pure flexural deformation, respectively. The results obtained are at variance with the static provisions of the current New Zealand code; this is especially so in the case of the shear wall. On the basis of these findings a tentative suggestion is made for a change to the familiar equivalent static lateral load distribution at present specified by the code.
\end{abstract}

\section{INTRODUCTION}

In a recent investigation of slender shear wall structures, Blakeley, Cooney and Megget (1) have shown that the base shear existing with a given base moment (say that at flexural capacity) may be substantially in excess of that specified by the equivalent static provisions of the code of practice NZS 4203 : 1976. This indicates that the lever arm of the dynamic forces may be considerably smaller than was originally thought possible. Their investigations included both linear elastic and inelastic dynamic studies.

The present paper involves the linear elastic analysis of a closely related problem, namely, the evaluation of likely positions for the centre of earthquake loading on slender high rise buildings.

\section{FORMULATION}

In this section a response spectrum technique due to Jennings $(2)$ is followed to derive expressions for the maximum likely base shear and maximum likely overturning moment for uniform continuous models of tall buildings responding linearly elastically to earthquake excitation. These quantities in themselves are not of interest for the present investigation. However, the ratio of this moment to this shear gives a likely position for the centre of earthquake loading at these maximum likely actions - which is the subject of the present study.

Under earthquake excitation the base shear is

$\mathrm{v}(0, t)=m \int_{0}^{l}|\ddot{z}(t)+\ddot{w}(x, t)| d x$,

and the overturning moment is

$M(0, t)=m \int_{0}^{l}|\ddot{z}(t)+\ddot{w}(x, t)| x d x$,

* Lecturer in Civil Engineering, University of Auckland. where $m$ is the (uniform) mass per unit length of the building, $l$ is its height, $\ddot{z}(t)$ is the base acceleration and $\ddot{w}(x, t)$ is the acceleration induced at height $x$ in the building relative to its base. Thus, $\{\ddot{z}(t)+\ddot{w}(x, t)\}$ is the absolute acceleration.

Now the relative displacement response of the building is

$w(x, t)=\sum_{n=1}^{\infty} \phi_{n}(x) \xi_{n}(t)$,

where $\phi_{n}(x)$ and $\xi_{n}(t)$ are the $n^{\text {th }}$ mode and $n^{\text {th }}$ normal coordinate of the equivalent continuous model, respectively. Because of this, and because of a well-known mathematical property of the modes, the absolute acceleration takes the alternative form

$|\ddot{z}(t)+\ddot{w}(x, t)|=\sum_{n=1}^{\infty}\left|\ddot{\xi}_{n}(t)+\alpha_{n} \ddot{z}(t)\right| \phi_{n}(x)$,

where $\alpha_{n}$ is the participation factor of the $\mathrm{n}$ th mode which, for a uniform cantilever, is given by

$\alpha_{n}=\int_{0}^{l} \phi_{n}(x) d x / \int_{0}^{l} \phi_{n}^{2}(x) d x$.

It is common to normalize the modes such that

$\int_{0}^{l} \phi_{n}^{2}(x) d x=l$

and this, torether with equations (4) and (5), gives

$v(0, t)=m \sum_{n=1}^{\infty}\left[\left|\ddot{\xi}_{n}(t)+\alpha_{n} \ddot{z}(t)\right| \quad \int_{0}^{l} \phi_{n}(x) d x\right]$,

and

$M(0, t)=\sum_{n=1}^{\infty}\left[\left|\ddot{\xi}_{n}(t)+\alpha_{n} \ddot{z}(t)\right| \int_{0}^{l} \phi_{n}(x) x d x\right]$. 
The maximum modal components are

$v_{n, \max }=m\left|\ddot{\xi}_{n}(t)+\alpha_{n} \ddot{z}(t)\right|_{\max } \int_{0}^{l} \phi_{n}(x) d x$.

and

$M_{n, \max }=m\left|\ddot{\xi}_{n}(t)+\alpha_{n} \ddot{z}(t)\right|_{\max } \int_{0}^{l} \phi_{n}(x) x d x$.

However

$\left|\ddot{\xi}_{n}(t)+\alpha_{n} \ddot{z}(t)\right|_{\max }=\alpha_{n} \omega_{n} S_{v n}$,

where $\omega_{n}$ is the natural circular frequency of vibration of the $n$th mode and $S_{y n}$ is the velocity spectrum value (3) relevant to the $n$th mode.

For the present it will be assumed that $S_{v n}$ is constant for all modes and equal to $\mathrm{S}_{\mathrm{V}}$. This is reasonable for the lower modes, but it tends to overestimate the higher modes - a matter which is taken up again in connection with the cantilever shear wall model. A further linked assumption is that damping is constant in the modes.

As a result of all this, it follows that the maximum likely base shear and overturning moment, which are simply the square roots of the sum of the squares of the maximum modal values given by equations (9) and (10) (a detailed discussion of this concept appears in the original paper by Goodman, Rosenblueth and Newmark (4), are

$v_{\max }=m S_{v}\left[\sum_{n=1}^{\infty}\left\{\left.\omega_{n}\left(\int_{0}^{l} \phi_{n}(x) d x\right)^{2}\right|^{2}\right]^{\frac{1}{2}}\right.$

and

$M_{\max }=m S_{v}\left[\sum_{n=1}^{\infty}\left|w_{n} \int_{0}^{l} \phi_{n}(x) d x \int_{0}^{l} \phi_{n}(x) x d x\right|^{2}\right]^{\frac{2}{2}}$.

The ratio $\mathrm{M}_{\max } / \mathrm{v}_{\max }$ then gives a likely position for the centre of earthquake loading - a result which, owing to the nature of the loading and the assumptions made, depends only on the fundamental dynamic properties of the model. While $M_{\text {max }}$ and $V_{\max }$ probably do not occur simultaneously, it is difficult to establish what the likely position of the centre is without a further considerable statistical effort. The ratio chosen seems reasonable, because different values of the ratio will be associated with lower values of one or other of the actions.

The theory is now applied to the cantilever shear beam and cantilever shear wall; these being two continuous models between which most uniform tall buildings will lie. First, however, the centre of earthquake loading is calculated according to the static provisions of the code.

\section{RESULT ACCORDING TO THE N.Z. CODE}

According to clause 3.4 .6 .1 of NZS 4203 : 1976, the centre of earthquake loading on a slender, uniform, tall building lies at a distance of

$\left\{\frac{2}{3} \times \frac{9}{10}+\frac{1}{10}\right\} \ell=0.7 \ell$

above its base. This equivalent static method is based on a procedure wherein $90 \%$ of the seismic loading is distributed up the building as a top-heavy triangle, while the remaining $10 \%$ is placed at the top (to simulate higher mode effects). The origins of the triangular load distribution can be traced to a detailed study of mainly first mode effects by a joint committee of californian engineers $(5)$.

\section{RESULT FOR CANTILEVER SHEAR BEAM}

It is well known that the natural frequencies and corresponding modes of the uniform cantilever shear beam are

$\left.\begin{array}{c}\omega_{n}=(2 n-1) c, \\ \phi_{n}(x)=\sqrt{2} \sin (2 n-1) \pi \frac{x}{l},\end{array}\right\}$

where $C$ is a dimensional constant for a given shear beam and $\phi_{n}(x)$ has been normalised according to equation (6). Consequently, $V_{\max }$ and $\mathrm{M}_{\max }$ are (2)

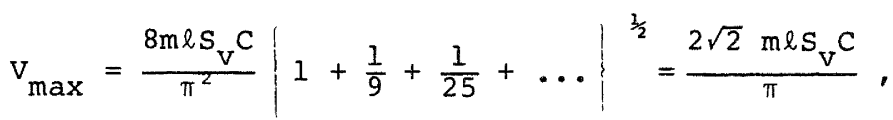

and

$M_{\max }=\frac{16 \mathrm{~m} \ell^{2} \mathrm{~S}_{V} \mathrm{C}}{\pi^{3}}\left|1+\frac{1}{81}+\frac{1}{625}+\ldots\right|^{\frac{1}{2}} \simeq \frac{16 \mathrm{~m} \ell^{2} \mathrm{~S}_{\mathrm{V}} \mathrm{C}}{\pi^{3}}$.

Therefore, a likely position for the centre of earthquake loading on the cantilever shear beam is at

$\frac{M_{\max }}{V_{\max }}=\frac{4 \sqrt{2} l}{\pi^{2}}=0.57 \ell$,

above the base. So far as base shear and overturning moment are concerned, the shear building "sees" only the first few modes and the assumptions regarding $\mathrm{S}_{\mathrm{V}}$ are not seriously called in question.

It may be noted that the resisting moments in the ground floor columns of a shear building depend on the shears in these columns and, therefore, apart from possibly important effects of axial forces in the columns, the centre of earthquake loading is not really a significant variable. Nevertheless, equation (17) does provide a result for one extreme case, and the reduction in column axial forces will be beneficial from the point of view of column interaction curves.

\section{RESULT FOR CANTILEVER SHEAR WALL}

It can be shown that the natural frequencies of the uniform cantilever shear wall are contained in the roots of the equation

$\cos \beta \ell \cosh \beta \ell=-1$. 
In particular, the natural frequencies are

$\omega_{n}=(\beta \ell)_{n}^{2} D, \quad n=1,2,3 \ldots$

where $D$ is a dimensional constant for a given shear wall and

$$
\left.\begin{array}{rl}
(\beta \ell)_{n} & =1.19 \frac{\pi}{2} \text { for } n=1, \\
& \simeq(2 n-1) \frac{\pi}{2} \text { for } n=2,3,4 \ldots .
\end{array}\right\}
$$

After some manipulation it may also be shown that the associated modes, normalised according to equation (6), are

$$
\begin{aligned}
\phi_{n}(x) & =\cosh \left\{(\beta l)_{n} \frac{x}{l}\right\}-\cos \left\{(\beta l)_{n} \frac{x}{l}\right\} \\
& -\left\{\frac{\cosh (\beta l)_{n}+\cos (\beta l)_{n}}{\sinh (\beta l)_{n}+\sin (\beta l)_{n}}\right\}\left[\sinh \left\{(\beta l)_{n} \frac{x}{l}\right)\right. \\
& -\sin \left\{(\beta \ell)_{n} \frac{x}{l}\right] .
\end{aligned}
$$

So, carrying out the necessary integrations (and making use of equations (18) and (19)) yields the following maximum likely modal components

$$
\left.v_{n, \max }=4 m \ell s_{v} D \mid \frac{\sinh (\beta \ell)_{n}-\sin (\beta \ell)_{n}}{\sinh (\beta \ell)_{n}+\sin (\beta \ell)_{n}}\right\} \text {, }
$$

and

$$
M_{n, \max }=4 \mathrm{ml}^{2} \mathrm{~S}_{\mathrm{v}} \mathrm{D} \frac{1}{(\beta \ell)_{n}}\left|\frac{\cosh (\beta \ell)_{n}+\cos (\beta \ell)_{n}}{\sinh (\beta l)_{n}+\sin (\beta \ell)_{n}}\right| .
$$

Therefore, the result for the maximum likely base shear is

$$
V_{\max }=4 \mathrm{mlS}_{\mathrm{V}} \mathrm{D} \quad\left\{(0.54)^{2}+(1.04)^{2}+1^{2}+\left.\ldots\right|^{\frac{1}{2}}\right. \text {, }
$$

while that for the maximum likely overturning moment is

$$
M_{\max }=\frac{8 m l^{2} S_{v} D}{\pi}\left\{(0.62)^{2}+(0.34)^{2}+\left(\frac{1}{5}\right)^{2}+\ldots\right\}^{\frac{1}{2}} .
$$

In contrast to the series for $M_{\max }$, the series for $V_{\max }$ is clearly divergent. This indicates that higher mode effects are of some importance for base shear in a shear wall although, naturally, a halt must be called somewhere.

Returning to equations (19) and (20), it is apparent that the natural frequencies of a shear wall increase very rapidly. In fact, they climb at a rate of approximately $(2 n-1)^{2}$ compared with $(2 n-1)$ for the shear beam. Therefore, what is called in question here is the validity of the assumption $\mathrm{S}_{\mathrm{Vn}}=\mathrm{S}_{\mathrm{V}}$ for all modes. This assumption quickly becomes untenable for the shear wall. At most, it could reasonably be expected that $V_{\max }$ consists of contributions from the first three modes, as outlined in equation (24).
The suggestion of further substantial contributions from modes higher than these, as implied by equation (22), is misleading because here $\mathrm{S}_{\mathrm{vn}} \rightarrow 0$.

Consequently, if just three modal components are considered, a likely position of the centre of earthquake loading is at

$\frac{M_{\max }}{V_{\max }}=0.30 \ell$

above the base. This compares quite strikingly with a value of 0.34 l for a ten storey wall obtained by Blakeley et al using an arbitrary superposition of the first three modes. On the other hand, if only two modes are considered *

$\frac{M_{\max }}{V_{\max }}=0.38 l$

For tall buildings responding elastically to earthquake excitation it is certainly reasonable to expect the presence of the first two modes at least. Thus, while equation (26) may perhaps represent a lower bound for the likely result (after all, it points to a bottom-heavy triangular block for the equivalent static loading distribution!), it is obvious that a wide gulf still exists between equation (27) and the value of $0.7 l$ derived from the code.

A method such as the trapezoidal rule may be used to replace equations (12) and (13) by discretized sums. Then this response spectrum method may be applied to problems where the natural frequencies and modes have been determined numerically. Of course, if the mass distribution varies up the wal1. equations (12) and (13) must be modified but, if only variations in stiffness are present, no changes are required. The first example treated by Blakeley et al was a 10 storey shear wall in which, although the floor masses were uniform, the flexural rigidity dropped linearly by a factor of two up the building. Using the data for the first two modes (only the relative values of which are of importance provided that they are normalised according to equation (6)) from their Figure 2 yields

$\frac{M_{\max }}{V_{\max }}=\frac{\left\{3.6^{2}+2.3^{2}\right\}^{\frac{1}{2}} l}{\left\{5.1^{2}+8.7^{2}\right\}^{\frac{1}{2}}}=0.42 \ell$.

The centre of earthquake loading is higher than that given by equation (27), and the reason for this most probably lies with the reduced stiffness at the upper floors - a point which is picked up again in the conclusions. A comparative study of different walls could well be based on an approach such as that outlined in this paragraph.

\section{DISCUSSION AND CONCLUSION}

Blakeley et al's elastic and inelastic analyses of cantilever shear walls have shown

* If only first mode responses are considered the values are $0.64 l$ and $0.73 l$ for the shear beam and shear wall, respectively. 
that the centres of earthquake loading frequently lie at positions which are substantially below that given by the code, and the present elastic study is in complete agreement with this. Thus, the base shear existing with a given overturning moment may be greatly in excess of that indicated by the code. Blakeley et al suggest a remedy whereby the shear envelope is increased by a factor ranging between 1.0 and 1.8 , depending on the height and class of the building. While this remedy certainly helps give a truer picture of the base shear likely to be present when the moment at the base of the wall reaches the level desired by the designer, it is by no means clear that the actual moment and shear requirements are not both overestimated by this process.

After all, if the likely position of the centre of earthquake loading on shear walls is as low as the present elastic study suggests, it would appear that it is the equivalent static distribution of lateral loading specified by the code that is in error. Rather than increase the shear, should not perhaps the moment be reduced by reducing its moment arm? (A result such as equation (24), which may argue for an increase in the basic seismic coefficient for shear walls, is here a side issue. As a separate topic, it could warrant further study.) Moves in this direction were made in several editions of the SEAOC code whereby the $J$ factor was invoked to reduce the overturning moment. However, the triangular distribution of lateral forces was not changed and $J$, and thus the reduction in moment, was strongly dependent on the fundamental period of the building - a feature which is not apparent in the present investigation. The J factor was dropped from the SEAOC code in the 1969 revision, although in a revised form it appears in the National Building Code of Canada : $1975(6)$.

In view of the present work it is hard to escape the conclusion that a lateral load uniformly distributed with height is what the New Zealand loading code should be recommending in its static provisions for uniform tall buildings. A centre of earthquake loading of $0.5 l$ would not be out of line with the present study. (It is ironic that a similar recommendation was part of one of the early New Zealand codes, namely, NZSS 95 : 1955.)

Against this it could be argued that a ductile cantilever shear wall yielding at its base is responding essentially as a rigid body rotating about its base. The appropriate centre of earthquake loading in this inelastic case would then be $0.667 \mathrm{l}$. (In fact, even before yield, the local weakening of the wall, associated with the cracking of the concrete at its base, will produce a condition in which this rigid body rotation is also a limiting case. Foundation rotation is also a factor to be contended with.) But, because the wall is not yielding all the way through its response, this is clearly an extreme situation and higher mode effects could be expected to lower the centre. Indeed, Blakeley et al's frequency distributions in their Figure 5 (b), (c) show that, for both a 15 storey wall and a 20 storey wall responding dynamically into the inelastic region, the most frequent positions lie between $0.55 l$ and $0.6 l$. However, the mean values of both distributions lie somewhat higher at about $0.65 l$.

Even so, the basis of comparision is suspect because the uniform shear wall of the present study is quite different from the more realistic examples of Blakeley et al where, although the mass distribution was uniform, the flexural rigidity decreased by a factor of two up the wall. This flexibility at higher levels may well raise the centre of earthquake loading.

To be sure, this recommendation of a change in the code lateral loading distribution is based solely on linear elastic theory applied to uniform buildings and, as such, must remain tentative pending further studies. Equally, however, while the suggested change has important ramifications, there is nothing sacred about the triangular distribution; if it is wrong it should be changed.

\section{ACKNOWLEDGEMENTS}

Useful suggestions and criticisms were made by N. A. Mowbray, R. Shepherd and R. C. Fenwick.

\section{REFERENCES}

1. Blakeley, R. W. G., Cooney, R. C., and Megget, L. M., "Seismic Shear Loading at Flexural Capacity in Cantilever wall Structures", Bulletin of the New Zealand National Society for Earthquake Engineering, Vol. 8, No. 4, December 1975, pp. $278-290$.

2. Jennings, P. C., "Spectrum Techniques for Tall Buildings", Proceedings of the Fourth World Conference on Earthquake Engineering, Santiago, Chile, Vol. 2, A-3, 1969, pp. 61-74.

3. Housner, G. W. , "Behaviour of structures During Earthquakes", Journal of the Engineering Mechanics Division, ASCE, Vol. 85, October 1959, pp. 109-129.

4. Goodman, L. E., Rosenblueth, E. and Newmark, N. M., "Aseismic Design of Firmly Founded Elastic Structures", Transactions of the American Society of Civil Engineers, Vol. 120, 1955, pp. 782-802.

5. Anderson, A. W., et al, "Lateral. Forces of Earthquake and Wind", Proceedings of the American Society of Civil Engineers, Vol. 77, April 1951, Separate No. 66.

6. Humar, J. L. and Wright, E. W., "Shear and Overturning Moment for EarthquakeResistant Building Design", Canadian Journal of Civil Engineering, Vol. 2, 1975, pp. 22-35. 